\title{
Using A3 Thinking to Improve the STAT Medication Process
}

\author{
Milisa Manojlovich, PhD, RN, CCRNN1,2*, Valerie J. Chase, MSE',3, Megan Mack, MD, \\ Meghan K. Conroy, RN, BSN, PCCN-CMC ${ }^{3}$, Karen Belanger, MS, RD 2,3, Debbie Zawol, MSN, RN, ACNS-BC ${ }^{2,3}$, \\ Karen M. Corr, PharmD ${ }^{3}$, Karen E. Fowler, MPH', Elizabeth Viglianti, MD, MPH ${ }^{3,4}$
}

${ }^{1}$ Division of Nursing Business and Health Systems, University of Michigan School of Nursing, Ann Arbor, Michigan; ${ }^{2}$ VA Ann Arbor Hospital Outcomes Program of Excellence (HOPE) Initiative, Ann Arbor, Michigan; ${ }^{3}$ VA Ann Arbor Healthcare System, Ann Arbor, Michigan; ${ }^{4}$ Department of Internal Medicine, University of Michigan Medical School, Ann Arbor, Michigan.

BACKGROUND: Although the term STAT conveys a sense of urgency, it is sometimes used to circumvent a system that may be too slow to accomplish tasks in a timely manner. We describe a quality-improvement project undertaken by a US Department of Veterans Affairs (VA) hospital to improve the STAT medication process.

METHODS: We adapted A3 Thinking, a problem-solving process common in Lean organizations, to our problem. In the discovery phase, a color-coded flow map of the existing process was constructed, and a real-time STAT order was followed in a modified "Go to the Gemba" exercise. In the envisioning phase, the team brainstormed to come up with as many improvement ideas as possible, which were then prioritized based on the anticipated effort and impact. The team then identified initial experiments to be carried out in the experimentation phase; each experiment followed a standard Plan-Do-Study-Act cycle.
RESULTS: On average, the number of STAT medications ordered per month decreased by $9.5 \%$. The average time from STAT order entry to administration decreased by $21 \%$, and time from medication delivery to administration decreased by $26 \%$. Improvements were also made in technician awareness of STAT medications and nurse notification of STAT medication delivery.

CONCLUSIONS: Adapting A3 Thinking for process improvement was a low-cost/low-tech option for a VA facility. The A3 Thinking process led to a better understanding of the meaning of STAT across disciplines, and promoted a collaborative culture in which other hospitalwide problems may be addressed in the future. Journal of Hospital Medicine 2014;9:540-544. 2014 Society of Hospital Medicine
STAT is an abbreviation of the Latin word statim, meaning immediately, ${ }^{1}$ and has been a part of healthcare's lexicon for almost as long as there have been hospitals. STAT conveys a sense of urgency, compelling those who hear STAT to act quickly. Unfortunately, given the lack of a consistent understanding of STAT, the term in reality often has an alternate use: to "hurry up" or to "complete sooner than routine," and is sometimes used to circumvent a system that is perceived to be too slow to accomplish a routine task in a timely manner.

As part of a larger systems redesign effort to improve patient safety and quality of care, an institutional review board (IRB)-approved qualitative study was conducted on 2 medical-surgical units in a US Department of Veterans Affairs (VA) hospital to

\footnotetext{
*Address for correspondence and reprint requests: Milisa Manojlovich, $\mathrm{PhD}$, Associate Professor, Division of Nursing Business and Health Systems, University of Michigan School of Nursing, 400 N Ingalls, Room 4306, Ann Arbor, Ml 48109-5482; Telephone: 734-936-3055; Fax: 734-6472416; E-mail: mmanojlo@umich.edu

Additional Supporting Information may be found in the online version of this article.

Published 2014. This article is a U.S. Government work and is in the public domain in the USA.

Received: January 22, 2014; Revised: April 1, 2014; Accepted: May 7, 2014

2014 Society of Hospital Medicine DOI 10.1002/jhm.2222

Published online in Wiley Online Library (Wileyonlinelibrary.com).
}

explore communication patterns between physicians and nurses. ${ }^{2}$ The study revealed wide variation in understanding between physicians and nurses on the ordering and administration of STAT medication. Physicians were unaware that when they placed a STAT order into the computerized patient record system (CPRS), nurses were not automatically alerted about the order. At this facility, nurses did not carry pagers. Although each unit had a supply of wireless telephones, they were often unreliable and therefore not used consistently. Nurses were required by policy to check the CPRS for new orders every 2 hours. This was an inefficient and possibly dangerous process, ${ }^{3}$ because if a nurse was not expecting a STAT order, 2 hours could elapse before she or he saw the order in the CPRS and began to look for the medication. A follow-up survey completed by physicians, nurses, pharmacists, and pharmacy technicians demonstrated stark differences on the definition of STAT and overlap with similar terms such as NOW and ASAP. Interviews with ordering providers indicated that $36 \%$ of the time a STAT was ordered it was not clinically urgent, but instead ordered STAT to "speed up the process."

The STAT medication process was clearly in need of improvement, but previous quality improvement projects in our organization had varying degrees of success. For example, we used Lean methodology in an attempt to improve our discharge process. We conducted a modified rapid process discharge 


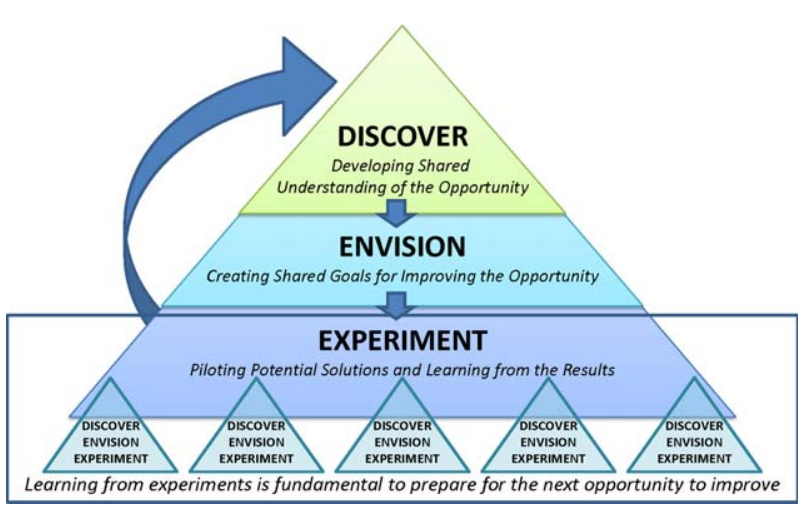

FIG. 1. Adaptation of the A3 Thinking conceptual model.

improvement workshop ${ }^{4}$ structured in phases over 4 weeks. During the workshops, a strong emphasis remained on the "solutions" to the "problem," and we were unable to help the team move from a mindset of "fix it" to "create it." This limited the buy-in of team members, the creativity of their ideas for improvement, and ultimately the momentum to improve the process.

In this article we describe our adaptation of A3 Thinking, ${ }^{5,6}$ a structure for guiding quality improvement based in Lean methodology, to improve the STAT medication process. We chose A3 Thinking for several reasons. A3 Thinking focuses on process improvement and thus aligned well with our interest in improving the STAT medication process. A3 Thinking also reveals otherwise hidden non-value-added activities that should be eliminated. ${ }^{7}$ Finally A3 Thinking reinforces a deeper understanding of the way the work is currently being done, providing critical information needed before making a change. This provides a tremendous opportunity to look at work differently and see opportunities for improvement. ${ }^{8}$ Given these strengths as well as the lack of congruence between what the STAT process should consist of and how the STAT process was actually being used in our organization, A3 Thinking offered the best fit between an improvement process and the problem to be solved.

\section{METHODS}

A search of healthcare literature yielded very few studies on the STAT process. ${ }^{9,10}$ Only 1 intervention to improve the process was found, and this focused on a specific procedure. ${ }^{10}$ An informal survey of local VA and non-VA hospitals regarding their experiences with the STAT medication process revealed insufficient information to aid our efforts. We next searched the business and manufacturing literature and found examples of how the Lean methodology was successfully applied to other problems in healthcare, including improving pediatric surgery workflow and decreasing ventilator-associated pneumonia. ${ }^{11,12}$

Therefore, the STAT project was structured to adapt a problem-solving process commonly used in
Lean organizations-A3 Thinking-which challenges team members to work through a discovery phase to develop a shared understanding of the process, an envisioning phase to conceptualize an ideal process experience, and finally an experimentation phase to identify and trial possible solutions through prioritization, iterative testing, structured reflection, and adjustment on resulting changes. Our application of the term experimentation in this context is distinct from that of controlled experimentation in clinical research; the term is intended to convey iterative learning as changes are tested, evaluated, and modified during this quality improvement project. Figure 1 displays a conceptual model of our adaptation of A3 Thinking. As this was a quality-improvement project, it was exempt from IRB review.

\section{DISCOVERY}

To begin the discovery phase, a workgroup consisting of representatives of all groups that had a role in the STAT process (ie, physician, pharmacist, nurse, pharmacy technician, clerk) gathered to identify "the opportunity we are looking to address" and learn from each other's individual experiences with the STAT medication process. The group was facilitated by an industrial engineer familiar with the A3 Thinking process. The team completed a mapping exercise to lay out, step-by-step, the current STAT medication process. This activity allowed the team to build shared empathy with others' experiences and to appreciate the challenges experienced by others through their individual responsibilities in the process. The current process was found to consist of 4 overarching components: a provider entered the STAT order into the CPRS; the order was verified by a pharmacist; a pharmacy technician delivered the medication to the unit (or a nurse retrieved the medication from the Omnicell (Omnicell Inc., Mountain View, CA), a proprietary automated medication dispensing system); and finally the nurse administered the medication to a patient.

A large, color-coded flow map of the STAT medication process was constructed over several meetings to capture all perspectives and allow team members to gather feedback from their peers. To further our understanding of the current process, the team participated in a modified "Go to the Gemba" (ie, go to where the work is done) ${ }^{13}$ on a real-time STAT order. Once all workgroup members were satisfied that the flow map represented the current state of the STAT medication process, we came to a consensus on the goals needed to meet our main objective.

We agreed that our main objective was that STAT medication orders should be recognized, verified, and administered to patients in a timely and appropriate manner to ensure quality care. We identified 3 goals to meet this objective: (1) STAT should be consistently defined and understood by everyone; (2) an easy, intuitive STAT process should be available for 
all stakeholders; and (3) the STAT process should be transparent and ideally visual so that everyone involved can understand at which point in the process a specific STAT order is currently situated. We also identified additional information we would need to reach the goals.

Shortly after the process-mapping sessions, 2 workgroup members conducted real-time STAT order time studies to track medications from order to administration. Three time periods in the STAT process were identified for observation and measurement: the time from physician order entry in the CPRS to the time a pharmacist verified the medication, the time from verification to when the medication arrived on the nursing unit, and the time from arrival on the nursing unit to when that medication was administered. Using a datacollection template, each time period was recorded, and 28 time studies were collected over 1 month. To monitor the progress of our initiatives, the time study was repeated 3 months into the project.

\section{ENVISIONING}

Following the discovery phase, the team was better equipped to identify the specific changes needed to achieve an improved process. The envisioning phase allowed the team freedom to imagine an ideal process barring any preconceived notion of constraints within the current process.

In 2 meetings we brainstormed as many improvement ideas as possible. To prioritize and focus our ideas, we developed a matrix (see Supporting Information, Appendix A, in the online version of this article), placing our ideas in 1 of 4 quadrants based on the anticipated effort to implement the change ( $\mathrm{x}$-axis) and impact of making the change (y-axis). The matrix helped us see that some ideas would be relatively simple to implement (eg, color-coded bags for STAT medication delivery), whereas others would require more sophisticated efforts and involvement of other people (eg, monthly education sessions to resident physicians).

\section{EXPERIMENTING}

Experiments were conducted to meet each of the 3 goals identified above. The team used the outcomes of the prioritization exercise to identify initial experiments to test. To build momentum by showing progress and improvement with a few quick wins, the team began with low-effort/high-impact opportunities. Each experiment followed a standard Plan-Do-StudyAct (PDSA) cycle to encourage reflection, learning, adaptation, and adjustment as a result of the experiential learning process. ${ }^{5}$

\section{Goal 1: STAT Should Be Consistently Defined and Understood by Everyone}

To address the first goal, a subgroup collected policies and procedures related to the STAT medication administration process. The policy defined a STAT medication as a medication that has the potential to significantly and negatively impact a patient's clinical condition if not given within 30 minutes. The group found that the policy requiring a 30-minute time to administration was clinically appropriate, reinforcing our goals to create a practice congruent with the policy.

A subgroup led by the pharmacy department collected data related to STAT medications on the 3 medical-surgical units. Within 1 month, 550 STAT medications were ordered, consisting of medications ranging from furosemide to nicotine lozenges, the latter being a medication clearly outside of the policy definition of STAT. The workgroup reviewed the information and realized education would be required to align practice with policy. According to our matrix, education was a high-impact/high-effort activity, so efforts were focused on the high-impact/low-effort activities initially. We addressed educational opportunities in later PDSA cycles.

\section{Goal 2: An Easy, Intuitive STAT Process for All Stakeholders}

The CPRS contains prefabricated templates that conform to regulatory requirements and ensure completeness. However, the CPRS does not intuitively enable ordering providers to choose the time for the first dose of a new routine medication. This often creates a situation where a provider orders the medication STAT, so that the medication can be given earlier than the CPRS would otherwise allow. Although there is a check box, "Give additional dose now," it was not being used because it was visually obscure in the interface. The CPRS restricted our ability to change the template for ordering medications to include a specific time for first-dose administration before defaulting to the routine order; thus, complementary countermeasures were trialed first. These are outlined in Table 1.

\section{Goal 3: The STAT Process Should Be Transparent and Ideally Visual}

During the time studies, the time period from when the medication arrived on the unit to the time it was administered to the patient averaged 34 minutes. Of 28 STAT orders followed through the entire process, 5 pharmacy technicians $(26 \%)$ were not informed of 19 STAT medication orders requiring delivery, and 12 nurses $(63 \%)$ were not notified of the delivery of those 19 medications. The remaining 9 STAT medications were stocked in the Omnicell. Informal interviews with nurses and pharmacy technicians, as well as input from the nurses and pharmacy technicians in our workgroup, revealed several explanations for these findings.

First, the delivering technicians could not always find the patient's nurse, and because the delivery procedure was not standardized, there was no consistency 
TABLE 1. Countermeasures Applied to Meet Goal 2

\begin{tabular}{ll}
\hline Countermeasure & \multicolumn{1}{c}{ Intended Outcome } \\
\hline Remove duplicate dosing frequencies from medication order template & Reduce list of dosing frequencies to sort through to find desired selection \\
Develop 1-page job aid for ordering providers to utilize & Assist in the correct methods of ordering STAT, NOW, and routine medications \\
Added "STAT ONCE" as a dosing frequency selection & Clarify the medication, if ordered STAT, will only be a 1-time administration to avoid the recurrence of a STAT \\
& order should the orders be transferred to a new unit with the patient \\
& Ensure documentation is congruent with new expectations \\
Modify existing policies to add "STAT ONCE" option & Inform ordering physicians on the available references for ordering and educate according to desired practice \\
Educate interns and residents with the job aid and a hands-on "how to" ordering exercise & In addition to providing information and educating according to desired practice, provide a just-in-time refer- \\
$\begin{array}{l}\text { Provide interns and residents with a visual job aid at their workstation and a } \\
\text { hands-on "how to" ordering exercise }\end{array}$ & ence resource
\end{tabular}

TABLE 2. Countermeasures Applied to Meet Goal 3

\begin{tabular}{lc}
\hline Countermeasure & Intended Outcome \\
\hline $\begin{array}{c}\text { Designate delivery preferences with the patient's nurse as the first } \\
\text { preference and a set location in the med room as the only alternative preference }\end{array}$ & Attempt to deliver medications directly to the patient's nurse as frequently as possible to eliminate \\
any unnecessary delays and avoid miscommunication \\
Identify a location in each unit's med room to place a red bin to deliver the STAT medications that are \\
unable to be delivered to the patient's nurse directly & $\begin{array}{c}\text { Provide } 1 \text { alternate location to retrieve STAT medications if the technician is unable to locate } \\
\text { the patient's nurse to deliver the medication directly }\end{array}$ \\
$\begin{array}{l}\text { Utilize a plastic bag with a red STAT indication for transportation of STAT medications to the units } \\
\text { Utilize red STAT magnets on the patient's door frame to signal nurses a medication had been }\end{array}$ & $\begin{array}{c}\text { Provide a visual to assist in pharmacy technicians prioritizing their deliveries to the inpatient units } \\
\text { delivered to the med room }\end{array}$ \\
\hline
\end{tabular}

between technicians in where medications were delivered. Second, each unit had a different medication inventory stored in the Omnicell, and the inventory was frequently changed (eg, due to unit-specific needs, backorders), which made it difficult for nurses to keep track of what was available in Omnicell at any given time. Finally, the STAT medication was not consistently labeled with a visual STAT notation, so even if a nurse saw that new medications had been delivered, he or she would not be able to easily identify which was STAT. The team made several low-tech process changes to improve the visibility of a STAT medication and ensure reliable communication upon delivery. A subgroup of pharmacists, technicians, and nurses developed and implemented the countermeasures described in Table 2.

\section{RESULTS}

At the start of our project, the average time from STAT order to medication administration was 1 hour and 7 minutes (range, 6 minutes -2 hours and 22 minutes). As a result of the 2 sets of countermeasures outlined in Tables 1 and 2, the average total time from STAT order entry to administration decreased by $21 \%$ to an average of 53 minutes. The total time from medication delivery to administration decreased by $26 \%$ from 34 minutes to 25 minutes postimplementation. On average, 391 STAT medications were ordered per month during the project period, which represents a decrease of $9.5 \%$ from the 432 orders per month for the same time period the previous year. After implementing the countermeasures in Table 2, we followed another 26 STAT medications through the process to evaluate our efforts. Of 15 STAT medications requiring delivery, only 1 nurse $(7 \%)$ was not notified of the delivery of a STAT medication, and 1 pharmacy technician $(7 \%)$ was not informed the medi- cation was STAT. The $151 \%$ increase in notification of nurses to delivery of a STAT medication suggests that use of the STAT bags, STAT magnets on patient doors, and whenever possible direct delivery of STAT medications to the nurse has improved communication between the technicians and nurses. Similarly, the $27 \%$ increase in technician awareness of a STAT designation suggests STAT is being better communicated to them. The improvement in awareness and notification of a STAT medication is summarized in Figure 2.

Due to time and financial constraints, the following limitations may have affected our findings. First, resident physicians were not directly represented in our discussions. Attending medicine hospitalists provided the physician perspective, which provides a biased view given their intimate knowledge of the CPRS and additional years of experience. Similarly, nurse perspectives were limited to staff and clinical nurse leaders. Last, our low-cost approach was mandated by limited resources; a more resource-rich environment may have devised alternative approaches.

\section{CONCLUSIONS}

Adapting A3 Thinking for process improvement was a low-cost/low-tech option for a VA facility. Having buy-in from all levels was crucial to the success of the project. The size and diversity of the group was also very important, as different opinions and aspects of the process were represented. Cross-discipline relationships and respect were formed, which will be valuable for collaboration in future projects. Although we focused on the STAT medication process, other quality-improvement projects could also benefit from A3 Thinking. Moreover, there were enough people to serve as ambassadors, taking the project back to their work areas to share with their peers, gather consensus, 


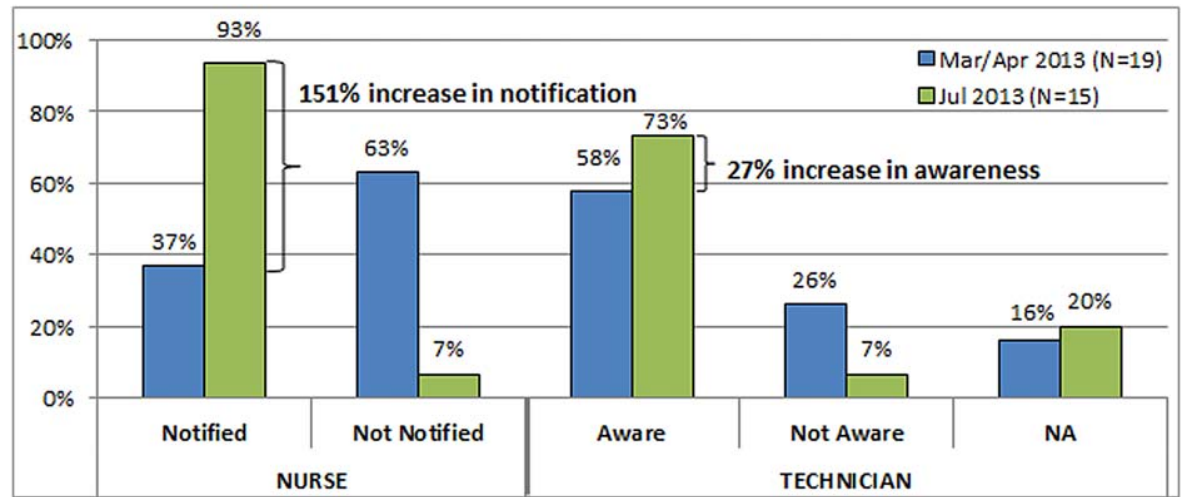

FIG. 2. Nurse and pharmacy technician notification/awareness of STAT medication. NA: there was no opportunity for technician awareness (eg, someone besides a pharmacy technician delivered the medication). Abbreviations: NA, not applicable.

and elicit additional feedback. The collaboration led to comprehensive understanding of the process, the nature of the problems within the process, and the complexity of solving the problem. For example, although the number of STAT orders did not decrease dramatically, we have learned from these experiments that we may need to change how we approach structuring additional experiments. Future work will focus on increasing communication between physicians and nurses when placing STAT medication orders, enhancing resident education to ensure appropriate use of the STAT designation, and continuing our efforts to improve the delivery process of STAT medications.

Other quality-improvement methodologies we could have used include: total quality management (TQM), continuous quality improvement (CQI), business process redesign, Lean, Six Sigma, and others. ${ }^{14}$ Differences between these can be broadly classified as putting an emphasis on people (eg, inclusion of front line staff in CQI or leadership in TQM) or on process (eg, understanding process function to reduce waste in Lean or statistical process control in Six Sigma). ${ }^{14}$ Using A3 Thinking methodology was more useful than these others for the STAT medication process for some very important reasons. The A3 process not only led to a better understanding of the meaning of STAT across disciplines, increasing the intuitive nature, transparency and visual aspects of the whole process, but also promoted a collaborative, multidisciplinary, integrative culture, in which other hospitalwide problems may be addressed in the future.

\section{Acknowledgements}

This work could not have been done without the contribution of all members of the STAT Improvement Workgroup, including Charles Alday; Allison Brenner, PharmD; Paula Carroll; Garry Davis; Michele Delaney, RN, MSN, CWCN; Mary East, MD; Stacy Frick, MSN, RN, CNL; Corry Gessner, CPhT; Kenya Harbin, MSN, RN, CNL; Crystal Heath, MS, RN-BC; Tom Kerr, MPH; Diane Klemer,
RPh; Diane Kohmescher, PharmD, BCPS; Sara Oberdick; Antanita Pickett; Ana Preda, CPhT; Joseph Pugh, RPh, MS; Gloria Salazar, CPhT; Samar Sheth, MD; Andrea Starnes, RN; Christine Wagner, PharmD; Leo Wallace; Roderick Williams; and Marilyn Woodruff.

Disclosures: This work was funded by a US Department of Veterans Affairs, Office of Systems Redesign Improvement Capability Grant and the Veterans in Partnership (VISN11) Healthcare Network. The findings and conclusions in this report are those of the authors and do not necessarily represent the position or policy of the US Department of Veterans Affairs. The authors have no other disclosures or conflicts to report.

\section{References}

1. The American Heritage Medical Dictionary of the English Language website . 2011. Available at: http://ahdictionary.com/word/search. html?q=STAT. Accessed December 22, 2013.

2. Manojlovich M, Harrod M, Holtz B, Hofer T, Kuhn L, Krein SL. The use of multiple qualitative methods to characterize communication events between physicians and nurses [published online ahead of print January 31, 2014]. Health Commun. doi: 10.1080/10410236.2013.835894.

3. Patterson ES, Rogers ML, Render ML. Fifteen best practice recommendations for bar-code medication administration in the Veterans Health Administration. Jt Comm J Qual Saf. 2004;30(7):355365 .

4. Womack JP, Byrne AP, Fiume OJ, Kaplan GS, Toussaint J. Going lean in health care. Cambridge, MA: Institute for Healthcare Improvement; 2005. Available at: http://www.ihi.org. Accessed March 19, 2014.

5. Sobek D, Smalley A. Understanding A3 Thinking: A Critical Component of Toyota's PDCA Management System. New York, NY: Productivity Press, Taylor \& Francis Group; 2008.

6. Shook J. Managing to Learn: Using the A3 Management Process to Solve Problems, Gain Agreement, Mentor and Lead. Cambridge, MA: Lean Enterprise Institute; 2008.

7. Varkey P, Reller MK, Resar RK. Basics of quality improvement in health care. Mayo Clin Proc. 2007;82(6):735-739.

8. Sobek DK, Jimmerson C. A3 problem solving: unique features of the A3 problem solving method. Available at: http://leanhealthcarewest. com/Page/A3-Problem-Solving. Accessed March 27, 2014.

9. Fahimi F, Sahraee Z, Amini S. Evaluation of stat orders in a teaching hospital: a chart review. Clin Drug Investig. 2011;31(4):231-235.

10. Wesp W. Using STAT properly. Radiol Manage. 2006;28(1):26-30; quiz 31-33.

11. Toussaint JS, Berry LL. The promise of Lean in health care. Mayo Clin Proc. 2013;88(1):74-82.

12. Kim CS, Spahlinger DA, Kin JM, Billi JE. Lean health care: what can hospitals learn from a world-class automaker? J Hosp Med. 2006; 1(3):191-199.

13. Imai M. Gemba Kaizen: A Commonsense Approach to a Continuous Improvement Strategy. 2nd ed. New York, NY: McGraw-Hill; 2012.

14. Walshe K. Pseudoinnovation: the development and spread of healthcare quality improvement methodologies. Int I Qual Health Care. 2009;21(3):153-159. 